\title{
Ortodoncia preventiva e interceptiva: Manejo de mordida abierta anterior y pérdida dental prematura.
}

\author{
Preventive and interceptive orthodontics: Management of anterior open bite \\ and premature loss of primary teeth.
}

Pamela Muñoz Cortés ${ }^{1 \mathrm{a}}$, M. Paulina SanPedro Bravo ${ }^{1 \mathrm{a}}$, Dayanne Mella Bustos ${ }^{2 b}$, Nicolás Páez Covarrubias ${ }^{2 b}$, José Antonio Plaza Santibáñez ${ }^{2 b}$.

\section{RESUMEN}

Antecedentes: El enfoque actual del tratamiento odontopediátrico, implica una visión holística con un manejo integral y multidisciplinario. Considera múltiples factores, bajo un enfoque de riesgo cariogénico con seguimiento del crecimiento y desarrollo craneofacial. Cuando hay compromiso funcional y estético se debe considerar un tratamiento preventivo, rehabilitador y de ortodoncia preventiva e interceptiva.

Presentación clínica: Niña de 9 años atendida en la Facultad de Odontología - Universidad de Chile. Al examen clínico presenta dentición mixta, en actividad de caries con alto riesgo cariogénico, neutroclusión molar y canina bilateral con mordida abierta anterior dentoalveolar por hábito de succión no nutritiva, dedo e interposición lingual en reposo y deglución. El tratamiento implicó la exodoncia prematura de un segundo molar primario; para conservar el espacio se colocó un mantenedor fijo de arco lingual como ortodoncia preventiva. Con el objetivo de evitar la interposición lingual, succión digital, recuperar el crecimiento transversal del maxilar y cerrar la mordida, se realizó ortodoncia interceptiva con un aparato ortopédico de expansión maxilar con rejilla palatina.

Relevancia clínica: El manejo terapéutico de las anomalías dentomaxilares es una competencia que puede ser realizada por el odontólogo general. Para el éxito es fundamental el diagnóstico precoz con una planificación clínica oportuna. Son tratamientos de resolución rápida y un factor clave es el control periódico.

Conclusión: En este caso clínico, el manejo del espacio dentario y la intervención temprana de la mordida abierta anterior mejoró la función y estética, junto al pronóstico de la anomalía dentomaxilar interceptable.
1. Departamento del Niño $y$ Ortopedia Dentomaxilar, Facultad de Odontología, Universidad de Chile, Santiago, Chile.

2. Facultad de Odontología, Universidad de Chile, Santiago, Chile.

a. DDS

b. Licenciado en Odontología.

Correspondencia:

Pamela Muñoz Cortés

Universidad de Chile. Facultad de Odontología. Dpto. del Niño y ODM. Olivos n 943 , Independencia,

Santiago, Chile.

pamelamunoz@odontologia.uchile.cl

PALABRAS CLAVES:

Odontología preventiva; Ortodoncia preventiva; Ortodoncia interceptiva; Mordida abierta; Mantenedor de espacio.

\section{KEYWORDS:}

Preventive dentistry; Preventive orthodontics; Interceptive orthodontics; Open bite; Space maintenance. 


\section{ABSTRACT}

Background: The current approach to pediatric dentistry treatment involves a holistic vision with a comprehensive and multidisciplinary management. It considers multiple factors under a cariogenic risk approach with monitoring of craniofacial growth and development. When there is functional and aesthetic commitment, preventive, restorative, and interceptive orthodontic treatment should be considered.

Clinical presentation: 9-year-old girl attended at the Faculty of Dentistry - Universidad de Chile. On clinical inspection she presented mixed dentition, caries activity with high cariogenic risk, bilateral molar and canine neutrocclusion with dentoalveolar anterior open bite due to nonnutritive suction habit, finger and lingual interposition in repose and deglutition. The treatment implied the premature extraction of a primary second molar; to preserve space a fixed lingual arch maintainer was placed as preventive orthodontics. In order to avoid lingual interposition, digital suction, recover maxillary transverse growth and close the bite; an interceptive orthodontics with a maxillary expander orthopedic with palatal grid was performed.

Clinical relevance: The therapeutic treatment of dentomaxillaries anomalies is a competence that can be performed by the general dentist. Early diagnosis with timely clinical planning is essential for success. They are rapid resolution treatments and periodic monitoring is a key factor.

Conclusion: In this clinical case, dental space management and early intervention of the anterior open bite improved function and aesthetic, as well as the prognosis of the

interceptable dentomaxillary anomaly.

\section{INTRODUCCIÓN}

El enfoque actual del tratamiento odontológico en niños y adolescentes implica una visión más amplia y holística, con un manejo integral y multidisciplinario, por lo que el tratamiento abarca el manejo de lesiones de caries y la evaluación del crecimiento y desarrollo craneofacial. El manejo terapéutico de manera oportuna y precoz puede ser conducido por el odontólogo general ${ }^{1}$. A nivel nacional y mundial las patologías orales más prevalentes en la población infantil son caries y anomalías dentomaxilares ${ }^{1-2}$. Hay que considerar diversos factores ambientales y funcionales asociados a las características dentofaciales del niño en crecimiento a partir de los primeros años de vida. La presencia de caries proximales en dientes primarios y sus complicaciones patológicas como la extracción prematura de estos, implica la pérdida de espacio para la erupción del diente permanente y se correlaciona con una mayor necesidad de tratamiento preventivo e interceptivo, para lo cual se recomienda el uso de mantenedor de espacio, existiendo diferentes guías clínicas y múltiples diseños. Su indicación va a depender de la situación clínica y el propósito, considerando su mantenimiento y control hasta finalizar la terapia ${ }^{3-5}$. Por otro lado, las presiones y fuerzas ambientales cambian la actividad estomatognática fisiológica $\mathrm{y}$ cualquier alteración en las funciones orales como deglución y fonoarticulación pueden influir en el crecimiento y desarrollo de las estructuras maxilofaciales ${ }^{3}$. Una de las anomalías dentomaxilares es la mordida abierta anterior, caracterizada por una relación vertical disminuida entre los bordes de los incisivos antagonistas cuando los molares están en oclusión. Es el resultado de un bloqueo mecánico del desarrollo vertical de los incisivos y del componente alveolar; a diferencia de la mordida abierta esqueletal que está determinada por una discrepancia esquelética vertical ${ }^{6}$. Numerosos hábitos e interacción de factores ambientales pueden provocar alteración en la oclusión y ser parte etiológica de esta patología; tales como componentes dentales, hábitos de succión prolongados, respiración bucal, empuje lingual o labial de los dientes anteriores ${ }^{6-8}$. En niños más pequeños, la presencia de mordida abierta anterior se asocia con hábitos orales no nutritivos y podría afectar negativamente su calidad de vida ${ }^{9}$. También la succión digital 
durante más de un año tiene mayor probabilidad de desarrollar anomalías en sentido sagital y vertical; con el consiguiente aumento del resalte y escalón ${ }^{10}$. Esta anomalía es corregible en pacientes de edades tempranas y en dentición mixta cuando se logra la normalización de la postura bucal, cierre labial, posición lingual y modificación del patrón de deglución, lo que genera cambios esqueléticos y dentomaxilares favorables ${ }^{3,8}$. El diagnóstico e intervención temprana de hábitos orales y factores ambientales es relevante en la prevención y reducción de la severidad de anomalías dentomaxilares en niños $9,11,12$.

\section{REPORTE DEL CASO}

\section{Información del paciente}

A mediados del año 2019, una paciente de 9 años acude de urgencia a atención odontopediátrica, por dolor en diente 8.5. En sus antecedentes personales presenta estrato social medio bajo, según índice de Graffar. Ha recibido atención odontológica por caries. Sin antecedentes mórbidos. Estado nutricional con índice de masa corporal normal. Se consigna el hábito de succión del pulgar izquierdo desde el año de vida. El apoderado firma el consentimiento informado según las normas de la Declaración de Helsinki y se informa de los protocolos de atención en la Clínica de la Facultad de Odontología Universidad de Chile.

\section{Hallazgos clínicos}

Al examen clínico extraoral presenta simetría facial vertical y horizontal, mesoprosopo, perfil anterior recto según análisis de Schwartz, rotación mandibular anterior, anteposición de cabeza y respiración oronasal. Al examen intraoral presenta dentición mixta segunda fase, poliobturaciones, múltiples lesiones de caries, gingivitis, mordida abierta anterior y asincronía de erupción dentaria del diente 1.2 (Fig 1). Al examen funcional se observa la lengua de tamaño normal con posición en reposo baja; en deglución, adelantada e interpuesta y en fonoarticulación con alteración de fonema "s". Como exámenes complementarios se toma radiografía retroalveolar del diente 8.5 , donde se observa un tratamiento compatible con pulpotomía y zona radiolúcida compatible con lesión osteolítica a nivel interradicular y perirradicular. Se registra un control radiográfico posterior a la exodoncia del diente 8.5. Para evaluar evolución de dentición y posición de caninos permanentes, se toma una ortopantomografía. Se observa presencia de gérmenes permanentes en evolución intra y extra ósea (Fig 2). Se realiza el análisis de Erikson y Kurol modificado por Lindauer para determinar el pronóstico de erupción espontánea, en base al desplazamiento e inclinación de los caninos permanentes superiores; clasificándose en sector I con pronóstico favorable. Al análisis de modelos en oclusión se registra en sentido sagital neutroclusión molar y canina bilateral, resalte de $2 \mathrm{~mm}$. En sentido vertical a nivel anterior un escalón de $-5 \mathrm{~mm}$. En sentido transversal líneas medias dentarias no coincidentes, con desviación inferior de 1 $\mathrm{mm}$. a la derecha con respecto a la línea media dentaria superior. Al análisis intramaxilar, se determina una compresión dentoalveolar leve por convergencia de los procesos alveolares hacia palatino.

\section{Evaluación del diagnóstico}

Paciente sexo femenino de 9 años, en riesgo social, dentición mixta segunda fase, en actividad de caries con alto riesgo cariogénico. Neutroclusión molar y canina bilateral, con mordida abierta anterior dentoalveolar por hábito no fisiológico de succión digital y deglución atípica por interposición lingual en reposo y alteración de la fonoarticulación. Sin riesgo de impactación canina superior y buen pronóstico de erupción. El pronóstico se consideró favorable, ya que se pueden manejar los factores de riesgo cariogénico realizando el tratamiento rehabilitador odontopediátrico. Además, presenta una anomalía dentomaxilar interceptable con un tratamiento de ortodoncia preventiva e interceptiva precoz en dentición mixta. La paciente y familia son cooperadores y comprometidos con el tratamiento. 

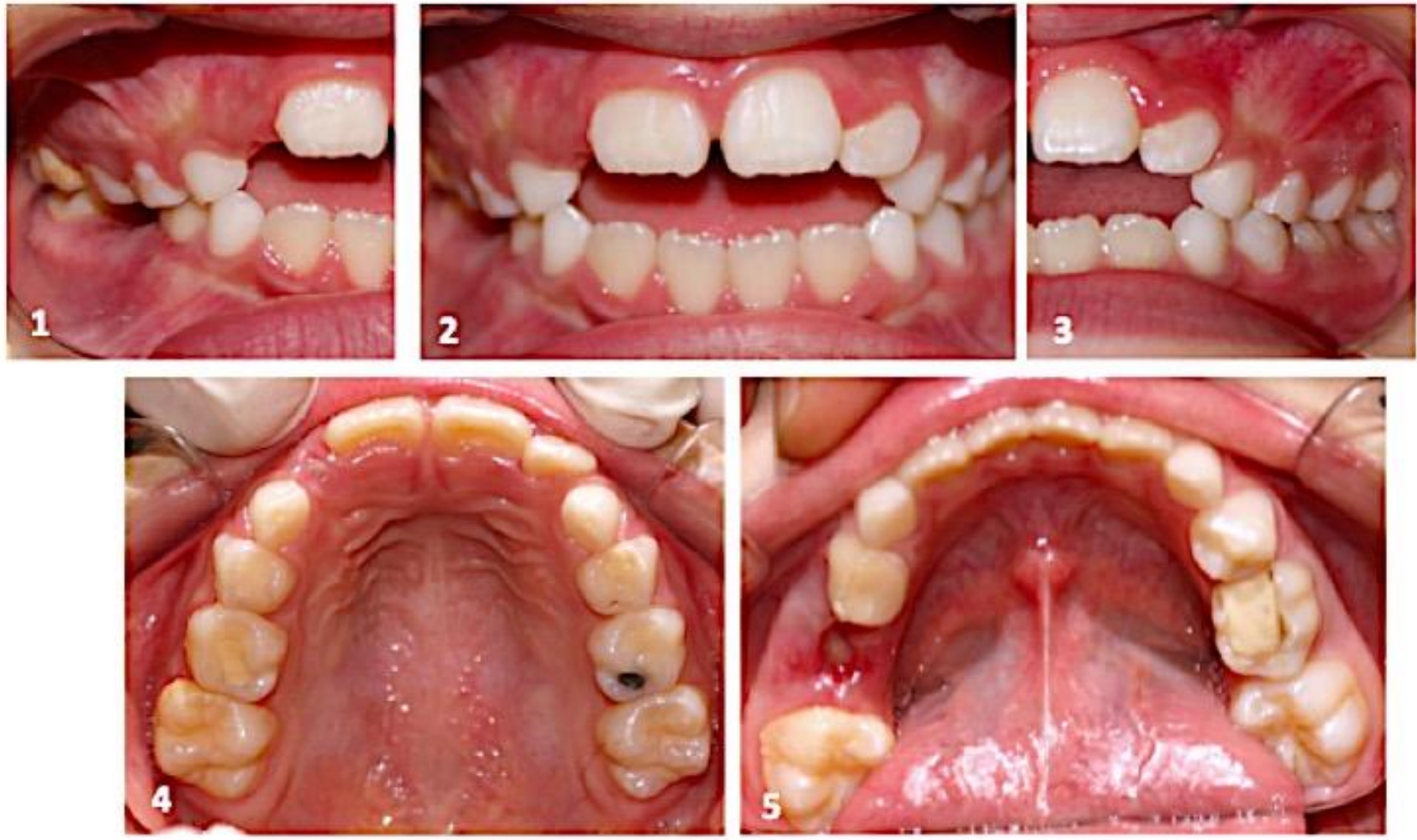

Figura 1: Registro de fotografías clínicas intraorales. (1) Vista lateral izquierda. (2) Vista frontal en oclusión. (3) Vista lateral derecha. (4) Vista oclusal de maxilar superior. (5) Vista oclusal de maxilar inferior.
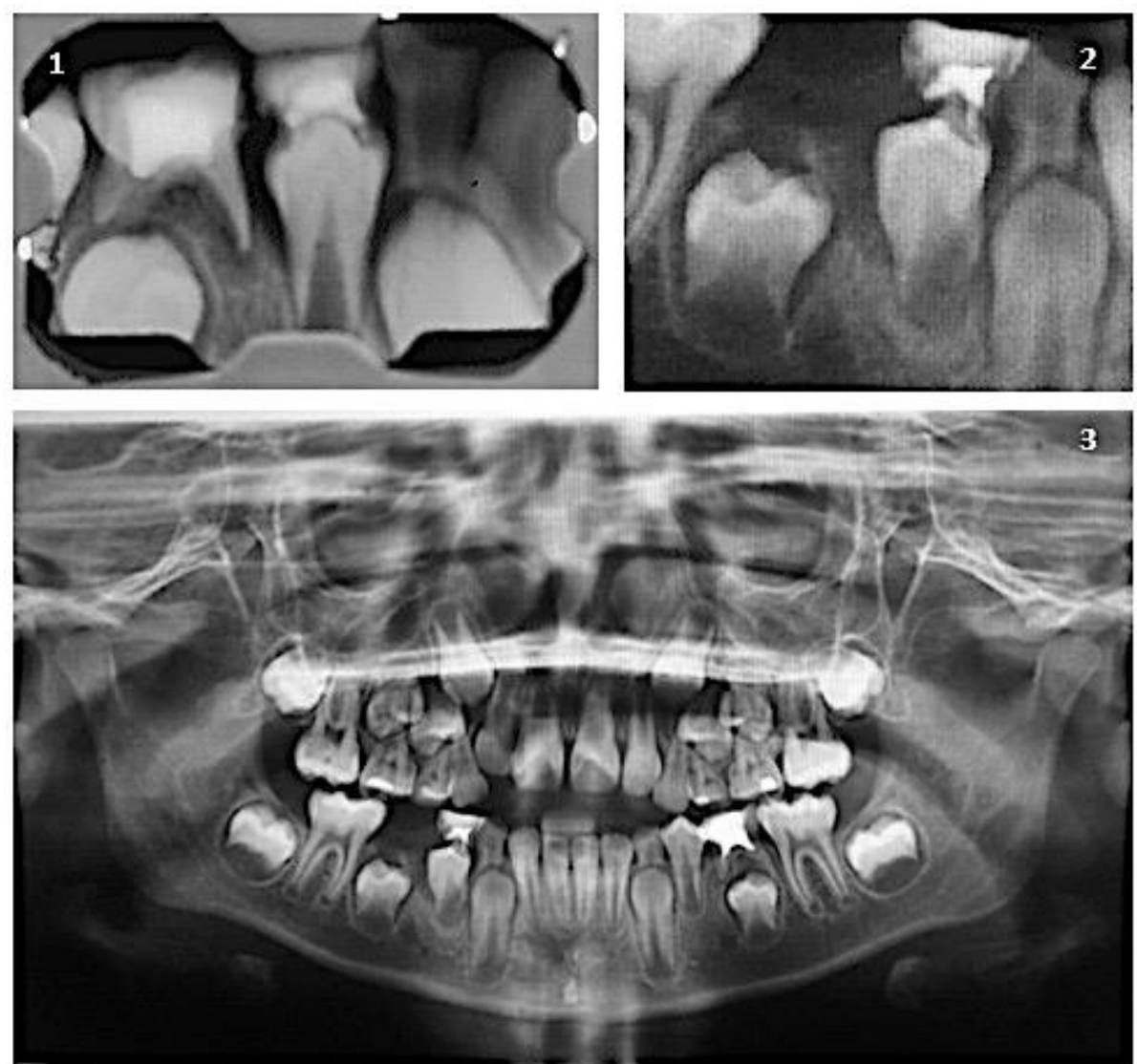

Figura 2: Registro radiográfico. (1) Radiografía retroalveolar diente 8.5. (2) Radiografía retroalveolar zona del diente 8.5 posterior a la exodoncia. (3) Ortopantomografia. 


\section{Intervención terapéutica}

$\mathrm{Al}$ existir un compromiso funcional $\mathrm{y}$ estético, se plantea un tratamiento multidisciplinario. Se realizó promoción de salud oral, prevención de caries, rehabilitación y exodoncia del diente 8.5. Con el objetivo de evitar una pérdida de espacio mesiodistal debido a la exodoncia prematura, se instaló un mantenedor de espacio fijo de arco lingual (Fig 3). Para el manejo de la mordida abierta anterior dentoalveolar se planificó un tratamiento de ortodoncia interceptiva. El objetivo fue recuperar el crecimiento transversal y vertical del maxilar, impedir la succión digital e interposición lingual. Se instaló un aparato ortopédico de expansión maxilar fijo, con tornillo de expansión central y rejilla palatina (Fig 3). Además, se motivó y se realizó refuerzo positivo a la paciente para que dejara gradualmente el hábito de succión digital.

\section{Seguimiento y resultados}

El tratamiento de ortodoncia interceptiva se indica por un periodo de 6 meses, sujeto a controles quincenales. El aparato expansor tuvo un régimen de activación lento por el odontólogo de 2 giros de $1 / 4$ de vuelta cada 2 semanas, durante 6 meses; con una expansión total de $6 \mathrm{~mm}$. A los 2 meses de seguimiento ya se observa un cierre parcial de la mordida abierta de 2 a $3 \mathrm{~mm}$. y cambios a nivel dentoalveolar en la inclinación de molares e incisivos evaluados clínicamente. A los 6 meses se evidencia el resultado estético esperado con un cierre completo de la mordida en anterior, presentando un escalón y resalte de $2 \mathrm{~mm}$. Se mantiene el aparato ortopédico en un periodo de contención de 4 meses y una vez retirado se deriva al kinesiólogo para la reeducación lingual con la finalidad de evitar la reinstauración de la interposición lingual. La rejilla palatina fue un elemento importante para impedir en esta primera etapa la interposición lingual y la succión digital (Fig. 4). Al control a los 6 meses de haber retirado el aparato, se evidencia que no hay recidiva (Fig. 4). Además, la paciente y su apoderado demostraron gran disposición a colaborar a lo largo del tratamiento. Se consideran controles posteriores para evaluar el desarrollo dentomaxilar y la evolución de los caninos permanentes para derivar oportunamente a ortodoncia en caso de que fuese necesario.

\section{DISCUSIÓN}

El tratamiento odontopediátrico debe ser integral y multidisciplinario, lo que requiere de competencias diagnósticas y clínicas por parte del odontólogo general; junto al manejo de tratamientos de ortodoncia preventiva $\mathrm{e}$ interceptiva, que tienen sus indicaciones y protocolos $^{5,10-12}$.
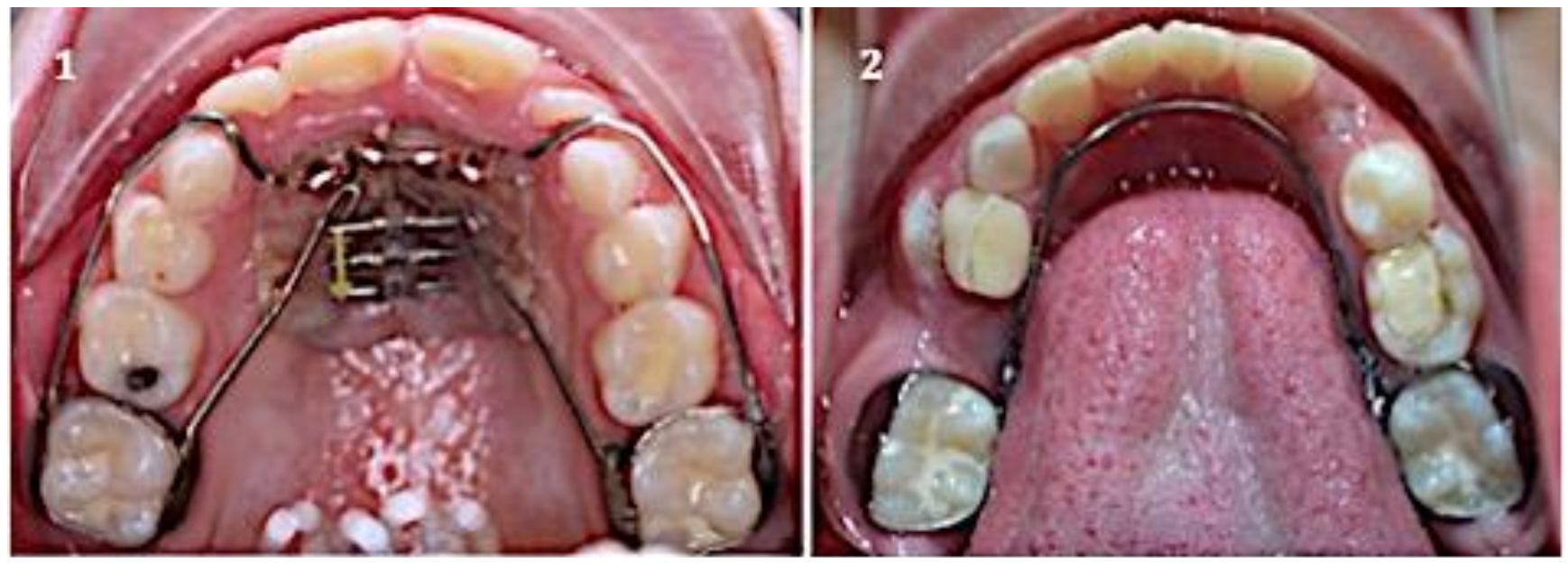

Figura 3: (1) Aparato de expansión maxilar fijo con tornillo medio y rejilla palatina. (2) Mantenedor de espacio fijo con arco lingual. 

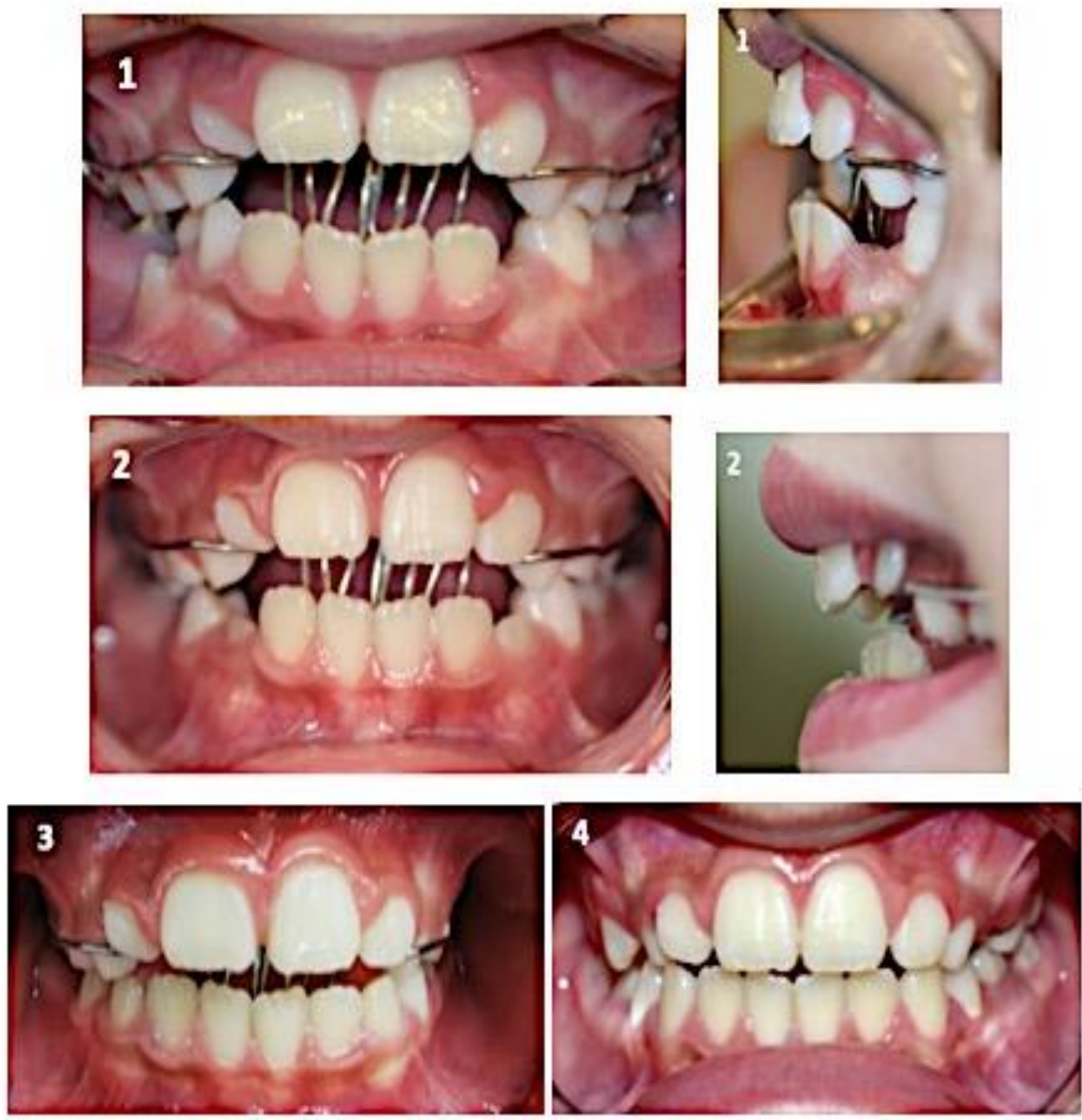

Figura 4: Registro fotográfico clínico de evolución del tratamiento de ortodoncia preventiva e interceptiva. (1) Instalación. Vista frontal y lateral. (2) Control a los 2 meses. Vista frontal y lateral. (3) Control a los 5 meses. Vista frontal. (4) Control a 6 meses de retirado el aparato. Vista frontal.

Estos tratamientos están indicados para intervenir el desarrollo de anomalías dentomaxilares de pacientes en dentición primaria o mixta y si la etiología es por factores ambientales, mejorará el pronóstico evitando una subsecuente alteración secundaria esqueletal y reducirá procedimientos más invasivos en el futuro ${ }^{4,6,11}$. En este caso clínico, el tratamiento de ortodoncia preventiva e interceptiva mejoró la función y estética. La derivación al kinesiólogo, una vez retirado el aparato ortopédico fue relevante para la reeducación lingual y evitar retomar los hábitos no fisiológicos que van a ocasionar recidiva y un daño potencial mayor ${ }^{9,10}$. Una de las principales ventajas es la resolución relativamente rápida y un factor clave es el control periódico, pues ante cualquier complicación o recidiva se deriva oportunamente al ortodoncista ${ }^{10}$. El manejo oportuno de la conservación del espacio dentario y la intervención temprana de anomalías dentomaxilares interceptables, podría recomendarse a nivel de atención primaria de salud y ser realizado por el odontólogo general. 


\section{CONFLICTOS DE INTERÉS}

Sin conflictos de interés

\section{REFERENCIAS}

[1] Cueto A, Skog F, Muñoz M, Espinoza S, Muñoz D, Martínez D. Prevalence of Dentomaxillary Anomalies and Treatment Needs in Adolescents. Int. J. Odontostomat. 2017 Sep; 11(3):333-8.

DOI:10.4067/S0718-381X2017000300333

[2] Fresno M. Consuelo, Jeldes Gabriela, Estay Juan, Martin Javier. Prevalencia, severidad de caries dental y necesidad de tratamiento restaurador en escolares de 6 a 12 Años de la Provincia de Santiago, Región Metropolitana. Rev. Clin. Periodoncia Implantol. Rehabil. Oral. 2019; 12(2): 81-6. DOI:10.4067/S0719-01072019000200081

[3] Rapepattana S, Suntornlohanakul S, Thearmontree A. Orthodontic treatment needs of children with high caries using Index for Preventive and Interceptive Orthodontic Needs (IPION). Eur Arch Paediatric Dent. 2019; 20(4):351-8. DOI:10.1007/s40368-019-00453-5

[4] Ahmad A, Parekh S, Ashley P. Methods of space maintenance for premature loss of a primary molar: a review. Eur Arch Paediatric Dent. 2018; 19(5):311-20.

DOI: $10.1007 / \mathrm{s} 40368-018-0357-5$

[5] Watt E, Ahmad A, Adamji R, Katsimbali A, Ashley $P$, Noar J. Space maintainers in the primary and mixed dentition - a clinical guide. Br Dent J. 2018;225(4):293-8.

DOI: 10.1038/sj.bdj.2018.650.

[6] Pisani L, Bonaccorso L, Fastuca R, Spena R, Lombardo L, Caprioglio A. Systematic review for orthodontic and orthopedic treatments for anterior open bite in the mixed dentition. Prog Orth. 2016;17(1).

DOI: 10.1186/s40510-016-0142-0

[7] Reichert I, Figel P, Winchester L. Orthodontic treatment of anterior open bite: a review article-is surgery always necessary? Oral and Maxillofacial Surgery. Oral Maxillofac Surg. 2014;18, 271-7.

DOI:10.1007/s10006-013-0430-5

[8] Koletsi D, Makou M, Pandis N. Effect of orthodontic management and orofacial muscle training protocols on the correction of myofunctional and myoskeletal problems in developing dentition. A systematic review and meta-analysis. Orthod Craniofac Res. 2018;21(4):202-15.

DOI: 10.1111/ocr.12240.

[9] Feres MF, Abreu LG, Insabralde NM, Almeida MR, Flores-Mir C. Effectiveness of the open bite treatment in growing children and adolescents. A systematic review. Eur J Orthod. 2016;38(3):237-50.

DOI: 10.1093/ejo/cjv048.

[10] Ling HTB, Sum FHKMH, Zhang L, Yeung CPW, Li KY, Wong HM, Yang Y. The association between nutritive, non-nutritive sucking habits and primary dental occlusion. BMC Oral Health. 2018; 22;18(1):145.

DOI: 10.1186/s12903-018-0610-7.

[11] Majorana A, Bardellini E, Amadori F, Conti G, Polimeni A. Timetable for oral prevention in childhood--developing dentition and oral habits: a current opinion. Prog Orthod. 2015; 16:39. DOI: 10.1186/s40510-015-0107-8.

[12] Sandoval Paulo, Bizcar Betty. Beneficios de la Implementación de Ortodoncia Interceptiva en la Clínica Infantil. Int. J. Odontostomat. 2013; 7(2): 253-65.

DOI:10.4067/S0718-381X2013000200016.

\section{CÓMO CITAR ESTE ARTÍCULO}

Muñoz P, San Pedro MP, Mella D, Páez N, Plaza JA Ortodoncia preventiva e interceptiva: Manejo de mordida abierta anterior y pérdida dental prematura. Appli Sci Dent. 2021:2(2); 42-8.

DOI: 10.22370/asd.2021.2.2.2536
Applied Sciences in Dentistry, revista científica de la Facultad de Odontología de la Universidad de Valparaíso, de Acceso Abierto y de Publicación Continua.

Son aceptados trabajos originales e inéditos, abarcando cartas al editor, comunicaciones breves, artículos de investigación, casos clínicos y revisiones bibliográficas.

Correo electrónico de contacto:

contacto.asdj@uv.cl

editor.asdj@uv.cl

Página Web:

https://revistas.uv.cl/index.php/asid

Redes Sociales

Instagram @asd.journal

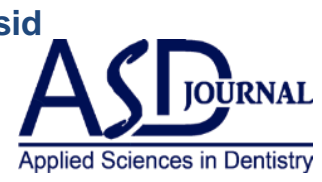

